\title{
CFAR Edge Detector for Polarimetric SAR Images
}

\section{Schou, Jesper; Skriver, Henning; Nielsen, Allan Aasbjerg; Conradsen, Knut}

\section{Published in:}

I E E E Transactions on Geoscience and Remote Sensing

Link to article, DOI:

10.1109/TGRS.2002.808063

Publication date:

2003

Document Version

Publisher's PDF, also known as Version of record

Link back to DTU Orbit

Citation (APA):

Schou, J., Skriver, H., Nielsen, A. A., \& Conradsen, K. (2003). CFAR Edge Detector for Polarimetric SAR Images. I E E E Transactions on Geoscience and Remote Sensing, 41(1), 20-32.

https://doi.org/10.1109/TGRS.2002.808063

\section{General rights}

Copyright and moral rights for the publications made accessible in the public portal are retained by the authors and/or other copyright owners and it is a condition of accessing publications that users recognise and abide by the legal requirements associated with these rights.

- Users may download and print one copy of any publication from the public portal for the purpose of private study or research.

- You may not further distribute the material or use it for any profit-making activity or commercial gain

- You may freely distribute the URL identifying the publication in the public portal 


\title{
CFAR Edge Detector for Polarimetric SAR Images
}

\author{
Jesper Schou, Henning Skriver, Allan Aasbjerg Nielsen, and Knut Conradsen
}

\begin{abstract}
Finding the edges between different regions in an image is one of the fundamental steps of image analysis, and several edge detectors suitable for the special statistics of synthetic aperture radar (SAR) intensity images have previously been developed. In this paper, a new edge detector for polarimetric SAR images is presented using a newly developed test statistic in the complex Wishart distribution to test for equality of covariance matrices. The new edge detector can be applied to a wide range of SAR data from single-channel intensity data to multifrequency and/or multitemporal polarimetric SAR data. By simply changing the parameters characterizing the test statistic according to the applied SAR data, constant false-alarm rate detection is always obtained. An adaptive filtering scheme is presented, and the distributions of the detector are verified using simulated polarimetric SAR images. Using SAR data from the Danish airborne polarimetric SAR, EMISAR, it is demonstrated that superior edge detection results are obtained using polarimetric and/or multifrequency data compared to using only intensity data.
\end{abstract}

Index Terms-Complex Wishart distribution, edge detection, polarimetry, synthetic aperture radar (SAR), Wishart likelihoodratio test statistic.

\section{INTRODUCTION}

$\mathbf{F}$ OR SINGLE-CHANNEL images, an edge is defined as an abrupt change in image intensity between two regions. Finding the edges in an image provides important information about the scene, and edge detection is often used as a preprocessing step in a given image analysis problem. An efficient edge detector should match the two criteria suggested in [1], i.e., it should determine the edge location accurately, and it should be robust in the sense that the probability of detecting an edge in a homogeneous region should be low, while actual edges need to be detected. The probability of detecting a nonexisting edge is normally denoted the probability of false alarms $P_{\mathrm{fa}}$.

In order to ensure a high robustness, the edge detector needs to be adapted to the image statistics. For intensity synthetic aperture radar (SAR) images, the observed intensity follows the well-known speckle distribution, i.e., the gamma distribution. Edge detectors suitable for optical images are thus not efficient for SAR images, as they result in the probabilities of false alarms being influenced by the mean intensities within the homogeneous regions [2], [3]. While edges in optical images are de-

\footnotetext{
Manuscript received June 12, 2001; revised October 17, 2002. This work was supported in part by The Danish National Research Councils under the ESA Follow-on Research Programme and under the Earth Observation Programme. The data acquisitions and the data preprocessing have been sponsored by the Danish National Research Foundation.

J. Schou and H. Skriver are with EMI, Section of Electromagnetic Systems, Technical University of Denmark (Ørsted DTU), DK-2800 Lyngby, Denmark (e-mail: hs@oersted.dtu.dk).

A. A. Nielsen and K. Conradsen are with IMM, Informatics and Mathematical Modelling, Technical University of Denmark (DTU), DK-2800 Lyngby, Denmark.

Digital Object Identifier 10.1109/TGRS.2002.808063
}

tected by finding the difference between two mean intensities on each side of the central pixel, the ratio edge detector suggested in [2] and [4] provides a constant false-alarm rate (CFAR) by taking the ratio of the same two means and this detector has been widely used by the SAR community [5]-[8].

Edge detection in SAR intensity images is also treated in [9], where it is shown that a single test can not fully optimize both performance criteria, i.e., a single detector can not provide the best edge-location determination and the highest possible probability of edge detection at the same time. CFAR-likelihood-ratio edge detectors are provided which optimize either of the two criteria and a combined edge detection scheme intended for optimizing both criteria is outlined. That work is extended in [10] by including texture in the treatment; hence, an edge is detected between regions having different mean intensity and/or texture. Also, Fjørtoft et al. [3] derive a CFAR edge detector based on the ratio of exponentially weighted means.

Polarimetric SAR data are completely described by the complex covariance matrix (CM) [11]. One approach to edge detection in polarimetric SAR images is to perform the detection separately for each polarization and subsequently combine the results using some kind of fusion operator [12], [13]. This approach, however, does not utilize the full polarimetric information, as only the backscattering coefficients of the CM are used. Also, the choice of fusion operator affects the final result. The detection can also be performed using a standard detector on a single image resulting from a combination of channels as it is donein [14].

In this paper, we describe a new approach to edge detection in polarimetric SAR data using a newly developed test for equality of complex covariance matrices following a complex Wishart distribution [15]. By estimating the CMs on each side of the central pixel, we test whether these two CMs are equal and edges are detected when the hypothesis is rejected. The new edge detector is a CFAR detector and it utilizes the full polarimetric information.

The paper is organized as follows. The complex Wishart distribution is described in Section II together with the test for equality of CMs. This test is used in Section III to derive the new polarimetric edge detector. The applied SAR data are discussed in Section IV, and the polarimetric edge detector is evaluated using simulated polarimetric SAR data as well as polarimetric EMISAR data in Section V. Section VI contains the conclusions.

\section{Image Statistics AND HyPothesis TeSt}

This section shows how multifrequency and/or multitemporal polarimetric SAR data are described using the complex Wishart distribution. This distribution is used in the test for equality of covariance matrices. 


\section{A. Complex Wishart Distribution}

A fully polarimetric SAR measures the $2 \times 2$ complex scattering matrix at each resolution cell on the ground. If $S_{i j}$ denotes the complex scattering amplitude for receive and transmit polarization $i$ and $j(i, j \in\{h, v\})$, then reciprocity, which normally applies to natural targets, gives $S_{\mathrm{hv}}=S_{\mathrm{vh}}$ (in the backscattering direction using the backscattering alignment convention) [11]. Assuming reciprocity, the scattering matrix is represented by the three-component complex target vector $\mathbf{s}=\left[\begin{array}{lll}S_{\mathrm{hh}} & S_{\mathrm{hv}} & S_{\mathrm{vv}}\end{array}\right]^{T}$, with $T$ being transpose, which follows a zero-mean multivariate Gaussian distribution [16]. The sample CM $\mathbf{C}$ is formed from the target vector as

$$
\begin{aligned}
\mathbf{C} & =4 \pi\left\langle\mathbf{s ~ s}^{* t}\right\rangle \\
& =\left[\begin{array}{ccc}
\sigma_{\mathrm{hh}} & \sqrt{\sigma_{\mathrm{hh}} \sigma_{\mathrm{hv}}} \rho_{\mathrm{hh}, \mathrm{hv}} & \sqrt{\sigma_{\mathrm{hh}} \sigma_{\mathrm{vv}}} \rho_{\mathrm{hh}, \mathrm{vv}} \\
\sqrt{\sigma_{\mathrm{hh}} \sigma_{\mathrm{hv}}} \rho_{\mathrm{hh}, \mathrm{hv}}^{*} & \sigma_{\mathrm{hv}} & \sqrt{\sigma_{\mathrm{hv}} \sigma_{\mathrm{vv}}} \rho_{\mathrm{hv}, \mathrm{vv}} \\
\sqrt{\sigma_{\mathrm{hh}} \sigma_{\mathrm{vv}}} \rho_{\mathrm{hh}, \mathrm{vv}}^{*} & \sqrt{\sigma_{\mathrm{hv}} \sigma_{\mathrm{vv}}} \rho_{\mathrm{hv}, \mathrm{vv}}^{*} & \sigma_{\mathrm{vv}}
\end{array}\right]
\end{aligned}
$$

where ${ }^{*}$ denotes complex conjugate; $\rho_{i, j}$ is the complex correlation coefficient between channels $i$ and $j$; and $\sigma$ is the backscattering coefficient.

If the $p \times p$ sample $\mathrm{CM}$ has $L$ number of looks, then we define the Hermitian matrix $\mathbf{Z}=L \mathbf{C}$. $\mathbf{Z}$ follows a complex Wishart distribution $W_{C}(p, L, \boldsymbol{\Sigma})$ having a mean CM $\Sigma$ [16], i.e.,

$$
p(\mathbf{Z} \mid \boldsymbol{\Sigma})=\frac{|\mathbf{Z}|^{L-p} e^{-\operatorname{tr}\left(\boldsymbol{\Sigma}^{-1} \mathbf{Z}\right)}}{\pi^{(1 / 2) p(p-1)} \prod_{j=1}^{p} \Gamma(L-j+1)|\boldsymbol{\Sigma}|^{n}}
$$

where $|\ldots|$ and $\operatorname{tr}(\ldots)$ denote the determinant and trace, respectively, and $\Gamma(\ldots)$ is the Gamma function. The mean CM provides information on the polarimetric properties of the surface. If the independent matrices $\mathbf{Z}_{1}, \ldots, \mathbf{Z}_{n}$ all follow the complex Wishart distribution having mean CM $\boldsymbol{\Sigma}$ and number of looks $L_{1}, \ldots, L_{n}$, respectively, then the sum of these $n$ matrices follows the complex Wishart distribution $W_{C}\left(p, L_{1}+\cdots+\right.$ $\left.L_{n}, \boldsymbol{\Sigma}\right)$. It should be mentioned that the distribution of the nonnormalized sample CM Z is presented in (2) rather than the distribution of the normalized sample $\mathrm{CM} \mathrm{C}$, since the test statistic applied in the following is based on $\mathbf{Z}$ [15].

The correlation between the copolarized and the cross-polarized terms (i.e., the absolute values of $\rho_{\mathrm{hh}, \mathrm{hv}}$ and $\rho_{\mathrm{hv}, \mathrm{vv}}$ ) often contain little information, and for randomly distributed targets with azimuthal symmetry the correlation is zero [17]. It is important to notice, however, that if the sample CM is forced into azimuthal symmetry (i.e., if the terms in the sample CM containing either $\rho_{\mathrm{hh}, \mathrm{hv}}$ or $\rho_{\mathrm{hv}, \mathrm{vv}}$ are set to zero), then the sample $\mathrm{CM}$ no longer follows the complex Wishart distribution [15]. In the following, we will encounter several examples where some of the elements of the sample CM have been set to zero, and we use the terms azimuthally symmetric (sample) CM, or the azimuthally symmetric case, for describing the case where $\rho_{\mathrm{hh}, \mathrm{hv}}$ and $\rho_{\mathrm{hv}, \mathrm{vv}}$ in the sample CM have been set to zero. Likewise, the terms diagonal (sample) CM, or the diagonal case, is used for describing the case where the off-diagonal components of the sample CM have been set to zero.

Multifrequency and/or multitemporal polarimetric data can also be described using the complex Wishart distribution. If $k$ is the number of polarimetric datasets acquired at different frequencies and/or acquisition times, and if the target vector data are available, the complex target vector is simply redefined as $\mathbf{s}=\left(\mathbf{s}_{1}^{T} \ldots \mathbf{s}_{k}^{T}\right)^{T}$ resulting in the sample CM $\mathbf{C}$

$$
\mathbf{C}=\left[\begin{array}{ccc}
\mathbf{C}_{1} & \cdots & \mathbf{C}_{1, k} \\
\vdots & \ddots & \vdots \\
\mathbf{C}_{k, 1} & \cdots & \mathbf{C}_{k}
\end{array}\right]
$$

where $\mathbf{C}_{i}$ is the sample $\mathrm{CM}$ for acquisition $i$, and the elements of the complex $p \times p$ matrices $\mathbf{C}_{i, j}, i \neq j$, depend on the correlation between the acquisitions $\left(\mathbf{C}_{i, j}=\mathbf{C}_{j, i}^{*}\right)$. Again, the $k p \times k p$ Hermitian matrix $\mathbf{Z}=L \mathbf{C}$ follows a complex Wishart distribution having $L$ number of looks. Often, however, the polarimetric SAR data from the $k$ acquisitions are already in the covariance matrix representation. In this case, the matrices $\mathbf{C}_{i, j}$ cannot be formed, and $\mathbf{Z}$ does not follow a complex Wishart distribution, as some of the elements of the covariance matrix have been set to zero.

\section{B. Test for Equality of Complex CM}

Conradsen et al. [15] have developed a test statistic in the complex Wishart distribution, where the likelihood-ratio test is used for testing the equality of two complex Wishart distributed matrices. For $\mathbf{Z}_{x}$ and $\mathbf{Z}_{y}$ following the complex Wishart distributions $W_{C}\left(p, n, \boldsymbol{\Sigma}_{x}\right)$ and $W_{C}\left(p, m, \boldsymbol{\Sigma}_{y}\right)$, respectively, we may examine if $\mathbf{Z}_{x}$ equals $\mathbf{Z}_{y}$ by considering the null hypothesis $H_{0}: \Sigma_{x}=\Sigma_{y}$, which states that the two matrices are equal against the alternative hypothesis $H_{1}: \boldsymbol{\Sigma}_{x} \neq \boldsymbol{\Sigma}_{y}$. The Wishart likelihood-ratio test statistic becomes [15]

$$
Q=\frac{(n+m)^{p(n+m)}}{n^{p n} m^{p m}} \cdot \frac{\left|\mathbf{Z}_{x}\right|^{n}\left|\mathbf{Z}_{y}\right|^{m}}{\left|\mathbf{Z}_{x}+\mathbf{Z}_{y}\right|^{n+m}}
$$

and the asymptotic distribution of $-2 \rho \log Q$ is given as

$$
\begin{aligned}
& P\{-2 \rho \log Q \leq z\} \simeq P\left\{\chi^{2}(f) \leq z\right\} \\
& \quad+\omega_{2}\left[P\left\{\chi^{2}(f+4) \leq z\right\}-P\left\{\chi^{2}(f) \leq z\right\}\right]
\end{aligned}
$$

with the parameters $f, \rho$, and $\omega_{2}$ defined as

$$
\begin{gathered}
f=p^{2} \\
\rho=1-\frac{2 p^{2}-1}{6 p}\left(\frac{1}{n}+\frac{1}{m}-\frac{1}{n+m}\right)
\end{gathered}
$$

and

$$
\begin{aligned}
& \omega_{2}=-\frac{f}{4}\left(1-\frac{1}{\rho}\right)^{2}+ \frac{f(f-1)}{24} \\
& \cdot\left(\frac{1}{n^{2}}+\frac{1}{m^{2}}-\frac{1}{(n+m)^{2}}\right) \frac{1}{\rho^{2}} .
\end{aligned}
$$

The term $-2 \rho \log Q$ is limited by [0, $\infty$, with $-2 \rho \log Q=0$ for $\mathbf{Z}_{x}=\mathbf{Z}_{y}$ and $n=m$.

As discussed in Section II-A, the sample CM is not complex Wishart distributed when one or more of the elements of the sample CM are set to zero. The test statistic described above is still applicable in these cases, however, when it is applied to the 
resulting submatrices [15]. In the following, this is demonstrated for the case of forced azimuthal symmetry, and the extension to the general case of multiple acquisitions is explained. The complex target vector can be redefined as

$$
\mathbf{s}=\left[\begin{array}{lll}
S_{\mathrm{hh}} & S_{\mathrm{vv}} & S_{\mathrm{hv}}
\end{array}\right]^{T}
$$

without introducing any constraints. The sample CM with forced azimuthal symmetry then becomes the block diagonal matrix

$$
\begin{aligned}
\mathbf{C} & =\left[\begin{array}{ccc}
\sigma_{\mathrm{hh}} & \sqrt{\sigma_{\mathrm{hh}} \sigma_{\mathrm{vv}}} \rho_{\mathrm{hh}, \mathrm{vv}} & 0 \\
\sqrt{\sigma_{\mathrm{hh}} \sigma_{\mathrm{vv}}} \rho_{\mathrm{hh}, \mathrm{vv}}^{*} & \sigma_{\mathrm{vv}} & 0 \\
0 & 0 & \sigma_{\mathrm{hv}}
\end{array}\right] \\
& =\left[\begin{array}{cc}
\mathbf{C}_{1} & \mathbf{0} \\
\mathbf{0}^{T} & \mathbf{C}_{2}
\end{array}\right]
\end{aligned}
$$

with $\mathbf{0}$ being the $2 \times 1$ null matrix, $\mathbf{C}_{1}$ the $2 \times 2$ sample $\mathrm{CM}$ for hh, and vv and $\mathbf{C}_{2}$ the $1 \times 1$ sample $\mathbf{C M}$ for hv. Using the same concepts as previously, we introduce the four mutually independent matrices $\mathbf{Z}_{x 1}, \mathbf{Z}_{x 2}, \mathbf{Z}_{y 1}$, and $\mathbf{Z}_{y 2}$ following the complex Wishart distributions

$$
\begin{aligned}
& \mathbf{Z}_{x 1}=n \mathbf{C}_{x 1} \in W_{C}\left(p_{1}, n, \boldsymbol{\Sigma}_{x 1}\right) \\
& \mathbf{Z}_{y 1}=m \mathbf{C}_{y 1} \in W_{C}\left(p_{1}, m, \boldsymbol{\Sigma}_{y 1}\right) \\
& \mathbf{Z}_{x 2}=n \mathbf{C}_{x 2} \in W_{C}\left(p_{2}, n, \boldsymbol{\Sigma}_{x 2}\right) \\
& \mathbf{Z}_{y 2}=m \mathbf{C}_{y 2} \in W_{C}\left(p_{2}, m, \boldsymbol{\Sigma}_{y 2}\right)
\end{aligned}
$$

with $p_{1}$ and $p_{2}$ being the dimension of $\mathbf{Z}_{x 1}\left(\mathbf{Z}_{y 1}\right)$ and $\mathbf{Z}_{x 2}\left(\mathbf{Z}_{y 2}\right)$, i.e., $p_{1}=2$ and $p_{2}=1$. The test for equality of the complex Wishart distributed matrices becomes [15]

$$
\begin{aligned}
H_{0}: \boldsymbol{\Sigma}_{x 1} & =\boldsymbol{\Sigma}_{y 1} \\
\boldsymbol{\Sigma}_{x 2} & =\boldsymbol{\Sigma}_{y 2}
\end{aligned}
$$

against all alternatives. The likelihood-ratio test statistic becomes [15]

$$
\begin{aligned}
Q= & \frac{(n+m)^{p_{1}(n+m)}}{n^{p_{1} n^{n}} m^{p_{1} m}} \cdot \frac{(n+m)^{p_{2}(n+m)}}{n^{p_{2} m^{p_{2} m}}} \\
& \cdot \frac{\left|\mathbf{Z}_{x 1}\right|^{n}\left|\mathbf{Z}_{y 1}\right|^{m}}{\left|\mathbf{Z}_{x 1}+\mathbf{Z}_{y 1}\right|^{n+m}} \cdot \frac{\left|\mathbf{Z}_{x 2}\right|^{n}\left|\mathbf{Z}_{y 2}\right|^{m}}{\left|\mathbf{Z}_{x 2}+\mathbf{Z}_{y 2}\right|^{n+m}} \\
= & \frac{(n+m)^{p(n+m)}}{n^{p n} m^{p m}} \cdot \frac{\left|\mathbf{Z}_{x}\right|^{n}\left|\mathbf{Z}_{y}\right|^{m}}{\left|\mathbf{Z}_{x}+\mathbf{Z}_{y}\right|^{n+m}}
\end{aligned}
$$

which is identical to the test statistic for the full covariance matrix (4). The asymptotic distribution of $-2 \rho \log Q$ is still given by (5), where the parameters $p, f, \rho$, and $\omega_{2}$ are now defined as

$$
\begin{gathered}
p^{2}=p_{1}^{2}+p_{2}^{2} \\
f=p^{2}=p_{1}^{2}+p_{2}^{2}=f_{1}+f_{2} \\
\rho=\frac{f_{1}}{f_{1}+f_{2}}\left(1-\frac{2 p_{1}^{2}-1}{6 p_{1}}\left(\frac{1}{n}+\frac{1}{m}-\frac{1}{n+m}\right)\right) \\
+\frac{f_{2}}{f_{1}+f_{2}}\left(1-\frac{2 p_{2}^{2}-1}{6 p_{2}}\left(\frac{1}{n}+\frac{1}{m}-\frac{1}{n+m}\right)\right) \\
=\frac{f_{1}}{f} \rho_{1}+\frac{f_{2}}{f} \rho_{2}
\end{gathered}
$$

and

$$
\begin{aligned}
\omega_{2}=-\frac{f}{4}\left(1-\frac{1}{\rho}\right)^{2} & +\frac{f_{1}\left(f_{1}-1\right)+f_{2}\left(f_{2}-1\right)}{24} \\
& \cdot\left(\frac{1}{n^{2}}+\frac{1}{m^{2}}-\frac{1}{(n+m)^{2}}\right) \frac{1}{\rho^{2}} .
\end{aligned}
$$

From (7)-(11d), it is seen that this approach can be extended directly to a wide combination of SAR data, and this demonstrates a very important characteristic of the Wishart likelihoodratio test statistic and the associated asymptotic distribution of $-2 \rho \log Q$. The test statistic can, for example, be applied directly to multiple acquisitions having different kind of information (some containing the full $3 \times 3$ sample CM, some containing the azimuthally symmetric sample CM, and some containing a single backscattering coefficient), and still the proper distribution of the test statistic can be derived. In the following notation, we always use $\mathbf{Z}_{x}$ and $\mathbf{Z}_{y}$ rather than writing the individual submatrices (e.g., $\mathbf{Z}_{x 1}, \mathbf{Z}_{x 2}, \mathbf{Z}_{y 1}$, and $\mathbf{Z}_{y 2}$ for the azimuthally symmetric case).

\section{EDGE DETECTION}

In this section, the hypothesis test described in the Section II is applied by the polarimetric edge detector. Also, the ratio edge detector is extended to utilize all three backscattering coefficients.

\section{A. Polarimetric CFAR Edge Detector}

The edge detection is performed by visiting each pixel in the image in succession and, for each pixel, applying a set of filters having different orientation. The filters estimate the mean covariance matrices on each side of the central pixel, and the test of equality of these two mean covariance matrices determines whether an edge is detected by the current filter or not. This scheme corresponds to the scanning-window, center-edge (SWCE) configuration in [9], which in the case of intensity SAR images provides optimum edge detection performance. To ensure the greatest amount of flexibility, the edge detection filters are defined according to Fig. 1. A filter configuration $K_{f}=\left\{l_{f}, w_{f}, d_{f}, \triangle v\right\}$ is described by the length, the width, the spacing of the filter, and the angular increment $\triangle v$ between two orientations ( $N_{f}=\pi / \triangle v$ filters exist for each configuration). The adaptive filtering scheme can now be described as follows.

1) Select first filter configuration.

2) For $N_{f}$ filters

a) Estimate $\mathbf{Z}_{x}$ and $\mathbf{Z}_{y}$ for filter $i$.

b) Calculate $Q_{(i)}$ using (4).

3) Find the minimum $Q_{(i)}, Q_{\min }$, of the $N_{f}$ values. $Q_{\min }$ corresponds to the maximum value of $-2 \rho \log Q$, $(-2 \rho \log Q)_{\max }$

4) If $(-2 \rho \log Q)_{\max }$ is larger than threshold an edge is detected. Save the orientation and strength of the edge; move to next pixel; and continue from Step 1).

5) Apply the next filter configuration, and continue from Step 2); or move to the next pixel, and continue from Step 1) if no more filter configurations are available. 


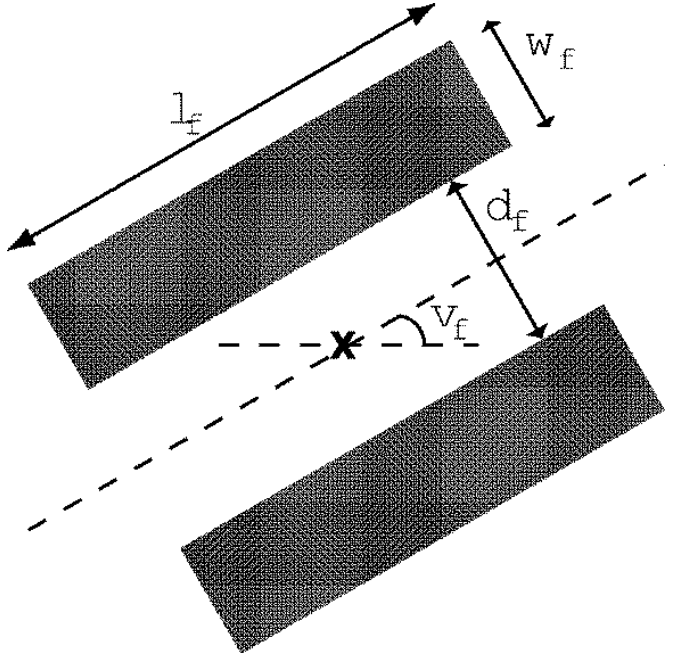

Fig. 1. Edge detection filter configuration $K_{f}=\left\{l_{f}, w_{f}, d_{f}, \Delta v\right\}$ is characterized by the following parameters. $l_{f}$ : length of the filter; $w_{f}$ : width of the edge regions; $d_{f}$ : spacing between the edge regions; and $\triangle v_{f}$ : angular increments between orientations $v_{i}$ and $v_{i+1} . N_{f}=\pi / \Delta v$ filters exist for each configuration.

When an edge is detected in Step 4) of the adaptive filtering scheme, the orientation (if needed) and edge strength $(-2 \rho \log Q)_{\max }$ of the specific filter are stored, and the next pixel is examined. If no edge is detected, the next filter configuration is applied. It should be noted that only the most significant edge is obtained from Step 4), since only $(-2 \rho \log Q)_{\max }$ is compared to the threshold; the individual $Q_{(i)}$ values should be compared to the threshold if the strengths and orientations of less significant edges are desired.

In Step 4) of the adaptive filtering scheme, it is tested whether $(-2 \rho \log Q)_{\max }$ is larger than a given threshold, and if this is the case, an edge is detected. The threshold depends on the number of looks for the two matrices, on the type of SAR data applied (e.g., fully polarimetric or azimuthally symmetric SAR data), and on the user-specified probability of false alarm $P_{\mathrm{fa}}$. The equivalent number of looks for $\mathbf{Z}_{x}$ and $\mathbf{Z}_{y}$ are equal, as they for a specific filter configuration are estimated from the same number of pixels, and in the following this number is denoted as $L_{f}$. If the pixels are independent, then $L_{f}=\left(w_{f} l_{f}\right) L$, but as this is generally not the case, we need to estimate $L_{f}$. This is done by averaging the backscatter images with a $w_{f} \times l_{f}$ window [2] and finding $L_{f}$ as the average number of looks of the three polarizations. Having estimated $L_{f}$, the threshold for the specific filter configuration $T_{f}$ is found. Detecting an edge corresponds to rejecting the null hypothesis, as the two mean covariance matrices are equal under the null hypothesis. The probability of rejecting the null hypothesis for a homogeneous region corresponds to the probability of false alarm; thus, we have

$$
P\left\{-2 \rho \log Q \leq T_{f}\right\}=1-P_{\text {fa }, 1}
$$

where $P_{\mathrm{fa}, 1}$ corresponds to the probability of false alarm for a single $Q$ value (i.e., $N_{f}=1$ ). In Step 3 ) of the adaptive filtering scheme, the minimum $Q$ is found from the $N_{f}$ stochastic vari- ables. $Q_{\min }$ corresponds to the maximum value of $-2 \rho \log Q$; hence, the distribution of $(-2 \rho \log Q)_{\max }$ is given by [18]

$$
P\left\{(-2 \rho \log Q)_{\max } \leq z\right\}=(P\{-2 \rho \log Q \leq z\})^{N_{f}}
$$

assuming independent variables $Q_{(i)}$. Combining (5), (12), and (13), we obtain the probability of false alarm for the $N_{f}$ filters $P_{\mathrm{fa}, N_{f}}$ as

$$
\begin{aligned}
P\left\{(-2 \rho \log Q)_{\max } \leq T_{f}\right\} \simeq & P\left\{\chi^{2}(f) \leq T_{f}\right\} \\
& +\omega_{2}\left[P\left\{\chi^{2}(f+4) \leq T_{f}\right\}\right. \\
& \left.\left.\quad-P\left\{\chi^{2}(f) \leq T_{f}\right\}\right]\right)^{N_{f}} \\
= & \left(1-P_{\mathrm{fa}, 1}\right)^{N_{f}} \\
\equiv & 1-P_{\mathrm{fa}, N_{f}} .
\end{aligned}
$$

For a given filter configuration and $P_{\mathrm{fa}, N_{f}},(14)$ is solved with regard to $T_{f}$, providing the specific threshold. Fig. 2 shows the relation between $P_{\mathrm{fa}, 1}$ and $T_{f}$ for the three cases of fully polarimetric CM data, forced azimuthally symmetric CM data, and forced diagonal CM data for $L_{f}=3$ and $L_{f}=13$. It is seen that $P_{\text {fa }, 1}$ is strongly dependent on $L_{f}$ for the fully polarimetric case, while only minor differences between the curves for $L_{f}=3$ and $L_{f}=13$ are observed for the other two cases. This is due to the fact that $\omega_{2}$ approaches zero quickly; for the diagonal sample $\mathrm{CM}$, it is practically zero for $L_{f}=3$; hence, the distribution (14) is simply a $\chi^{2}$-distribution having $f=3$ degrees of freedom.

In Fig. 2, it is seen that the thresholds are approximately identical for $L_{f}=3$ and $L_{f}=13$ (at least for the azimuthally symmetric and diagonal case), but the test statistic is strongly dependent on the equivalent number of looks. This can be demonstrated using $\mathbf{Z}_{y}=k_{y} \mathbf{Z}_{x}$ and $m=n$ (i.e., the same equivalent number of looks for the two matrices), in which case the Wishart likelihood-ratio test statistic (4) becomes

$$
Q=2^{2 p n}\left(\frac{\left|\mathbf{Z}_{x}\right|\left|k_{y} \mathbf{Z}_{x}\right|}{\left|\left(1+k_{y}\right) \mathbf{Z}_{x}\right|^{2}}\right)^{n}=2^{2 p n} \frac{k_{y}^{p n}}{\left(1+k_{y}\right)^{2 p n}} .
$$

Fig. 3 shows two plots of $-2 \rho \log Q$ using (15) as a function of $n$ and using $k_{y}$ equal to two and five, respectively. From the plots, it is seen that increasing $n$ values and increasing $k_{y}$ values both result in increasing values of $-2 \rho \log Q$. This means that a large test statistic is obtained (i.e., an edge is detected) from two sample covariance matrices $\mathbf{Z}_{x}$ and $\mathbf{Z}_{y}$, which are either significantly different or less different but representing a high equivalent number of looks.

This procedure assumes independent $Q_{(i)}$ variables, but the variables are not independent, as the neighborhoods from which $\mathbf{Z}_{x}$ and $\mathbf{Z}_{y}$ are estimated are overlapping between filters [2]. In Section V-A, it is shown how changing the number of variables in (14), $N_{f}$ provides a reasonable match between the theoretical distribution and the observations.

The asymptotic distribution of $-2 \rho \log Q$ in (5) only depends on the dimension of the CM and the number of looks for the two 


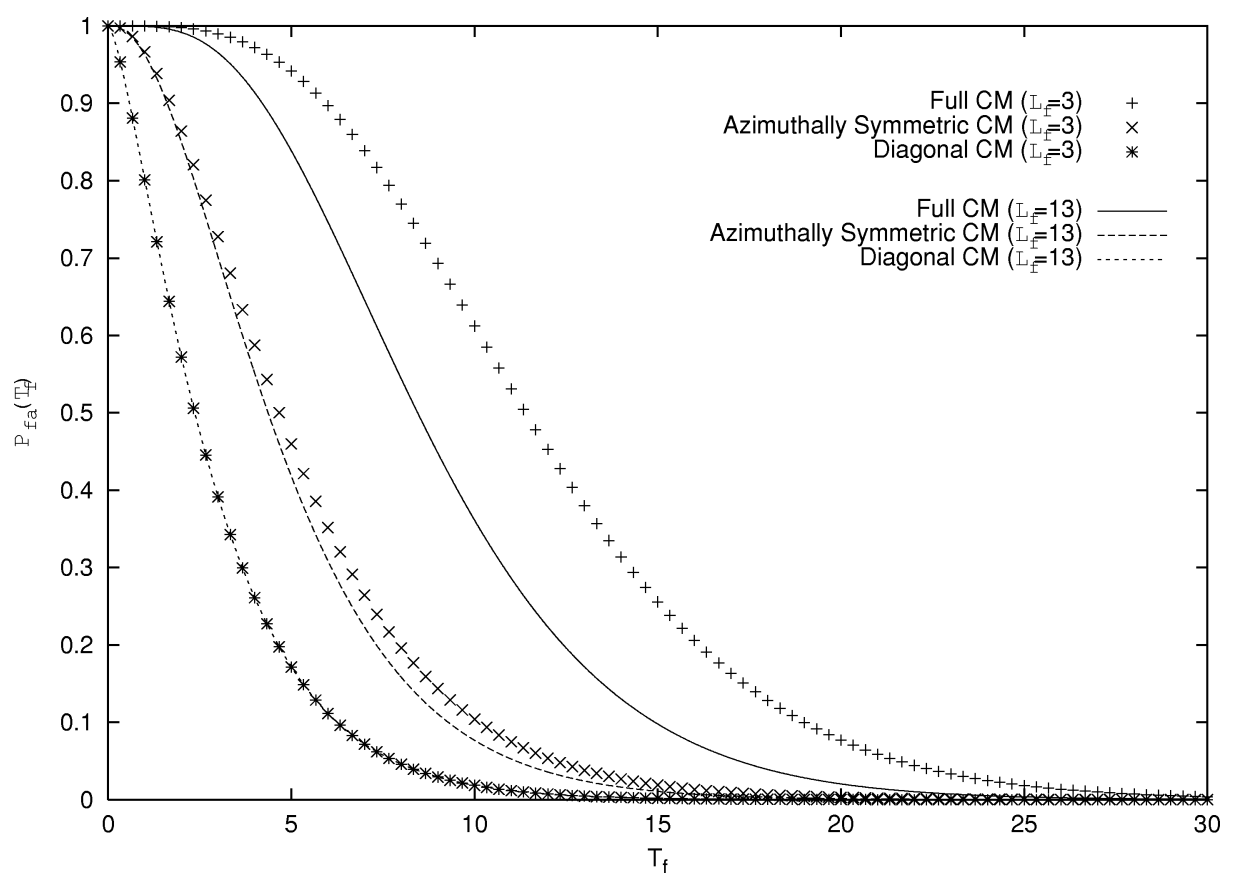

Fig. 2. Probability of false alarm as a function of the threshold for the case of full $\mathrm{CM}$ data, azimuthally symmetric $\mathrm{CM}$ data, and diagonal $\mathrm{CM}$ data for $L_{f}=3$ and $L_{f}=13$.

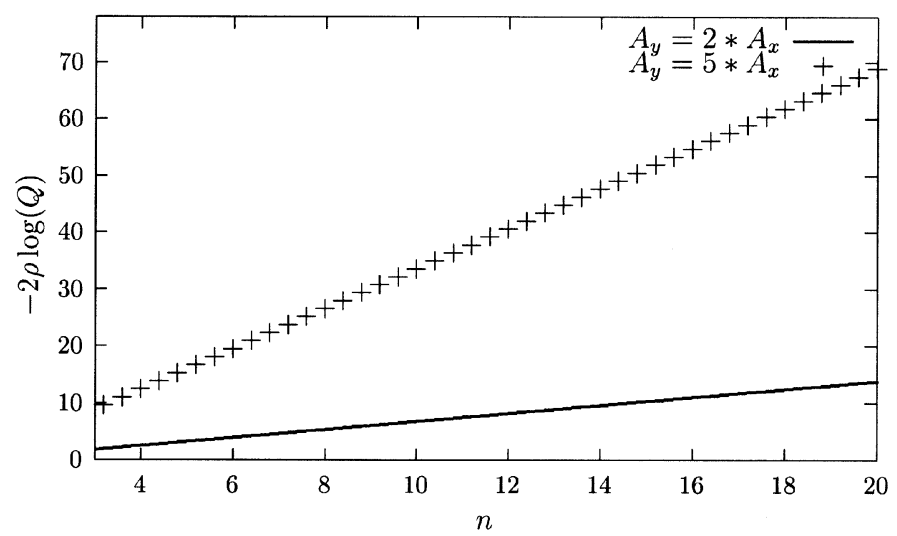

Fig. 3. Test statistic $-2 \rho \log Q$ as a function of $n$ (equivalent number of looks) using $m=n$ and $\mathbf{Z}_{y}=k_{y} \mathbf{Z}_{x}, k_{y}=2$, and 5, respectively.

matrices $\mathbf{Z}_{x}$ and $\mathbf{Z}_{y}$, as seen in (6a)-(6c) for the standard polarimetric case and (11a)-(11d) for the azimuthally symmetric case. In (14), it is shown that the asymptotic distribution is directly related to $P_{\mathrm{fa}, N_{f}}$, i.e., the probability of false alarm is independent of the content of the CM (e.g., the backscattering levels), and hence a constant false-alarm rate is obtained for all homogeneous regions in the scene by using the same threshold $T_{f}$. Therefore, the new polarimetric detector belongs to the group of CFAR detectors, which also includes the standard ratio edge detector [2], [4].

\section{B. Ratio Edge Detector}

Using an edge detection scheme similar to the one described in Section III-A, the ratio edge detector [2], [4] estimates the mean intensities on each side of the central pixel and detects edges based on the ratio of these intensities. If the estimated intensities are denoted $I_{x}$ and $I_{y}$, the ratio $r$ is defined as

$$
r=\min \left(\frac{I_{x}}{I_{y}}, \frac{I_{y}}{I_{x}}\right)
$$

resulting in small values of $r$ for edges having a large contrast. A direct comparison between the ratio detector and the polarimetric detector is difficult, as the ratio detector only applies to single polarization images. Using a fusion operator in combination with the ratio detector makes it possible to apply multiple intensity images, allowing for a better comparison with the polarimetric detector using diagonal CM data. In the following, the $\min (\ldots)$ operator is applied, and the filtering scheme follows the scheme from the polarimetric detector described in Section III-A, except it is the ratio $r_{(i)}$ for filter $i$ that is calculated in Step 2). Also, $N_{f}$ filters are applied for each of the $n$ images ( $n=3$ when the three backscatter images are applied). In Step 3) of the scheme, the minimum $r_{(i)}, r_{\min }$, is chosen, and if this value is smaller than a given threshold, then an edge is detected in Step 4). Applying the ratio detector to $n$ backscatter images results in an effective number of filters equal to $n \times N_{f}$; hence, the distribution of $r_{\min }$ is [18]

$$
P\left\{r_{\min } \leq z\right\}=1-(1-P\{r \leq z\})^{n \times N_{f}}
$$

assuming independent variables $r_{(i)}$. The threshold corresponding to a given filter configuration and probability of false alarm is found using the procedure in [2]. However, as for the polarimetric edge detector, the variables are correlated; hence, the number of independent variables in (17) is smaller, as discussed in Section V-B. 


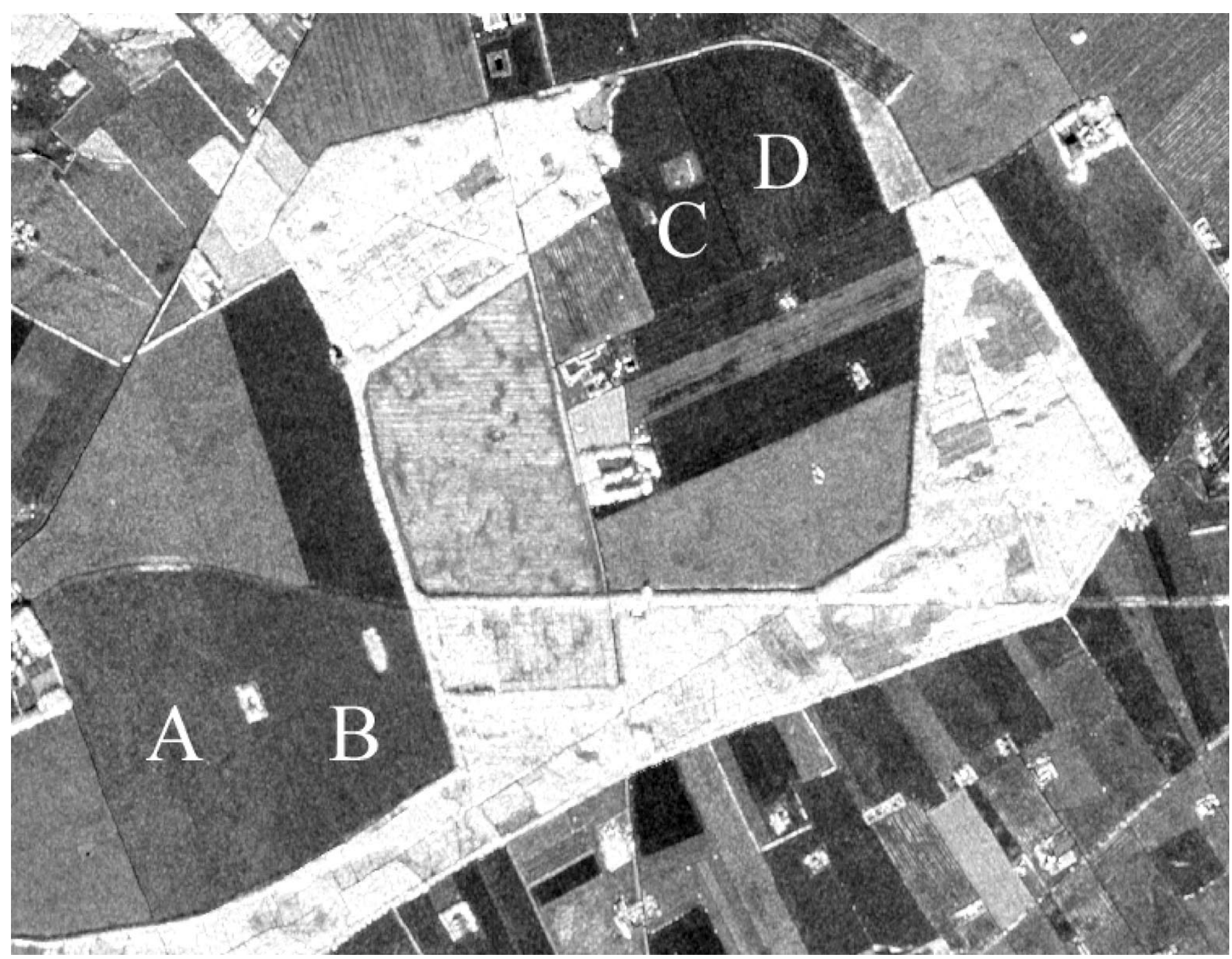

Fig. 4. EMISAR Span-image of test area, L-band May 20. The four fields are (A) winter wheat, (B) spring barley, (C) oats, and (D) peas.

The ratio detector, using single or multiple polarization images, results in values in the interval $] 0,1]$ with small values corresponding to strong edges. In the figures shown in Section V, the ratio values are inverted to appear like the results from the polarimetric detector; hence, an edge appears bright in the edge detection results (the edge maps) from both detectors.

\section{SAR DATA}

We apply L- and C-band EMISAR data as well as simulated L- and C-band SAR data in this paper.

\section{A. EMISAR Data}

The Danish airborne EMISAR system is a fully polarimetric SAR operating at L- and C-band $(1.25$ and $5.3 \mathrm{GHz}$, respectively) [19]. EMISAR is developed and operated at the Section of Electromagnetic Systems, Ørsted Department, Technical University of Denmark (DTU) and flown on board a Gulfstream G-3 aircraft from the Royal Danish Air Force at an altitude of typically $12.5 \mathrm{~km}$. The images applied in this paper are L- and C-band 13-look covariance matrix data at a $5 \times 5 \mathrm{~m}$ ground pixel spacing, having a spatial resolution of approximately 8 $\mathrm{m}$. The datasets are geometrically rectified resulting in subpixel root mean-square accuracy [20]. Fig. 4 shows a Span-image, i.e., $\sigma_{\mathrm{hh}}+2 \sigma_{\mathrm{hv}}+\sigma_{\mathrm{vv}}$, of the test area near Research Centre Foulum, Denmark, containing a number of agricultural fields, farms, hedges, and forest areas.

\section{B. Simulated SAR Data}

Applying simulated data provides an efficient way of evaluating image analysis algorithms. In the case of edge detection, simulated images provide information of the exact location of the edges, as well as information of the different classes within the images. The Potts model [21] is used for generating a test image having seven classes, as seen in Fig. 5(a), and in the following, this test image is denoted as the "cartoon image." Scattering matrix data are generated from the cartoon image using the approach suggested in [22], although we generate homogeneous data rather than apply the $K$-distribution. The scattering matrix data are transformed to 13-look covariance matrix data using the same processing scheme applied to real EMISAR data, i.e., a cosine-squared weighted $9 \times 9$ filter. To ensure the highest degree of consistency with actual EMISAR data, the covariance matrix parameters for the seven classes reflect the parameters 


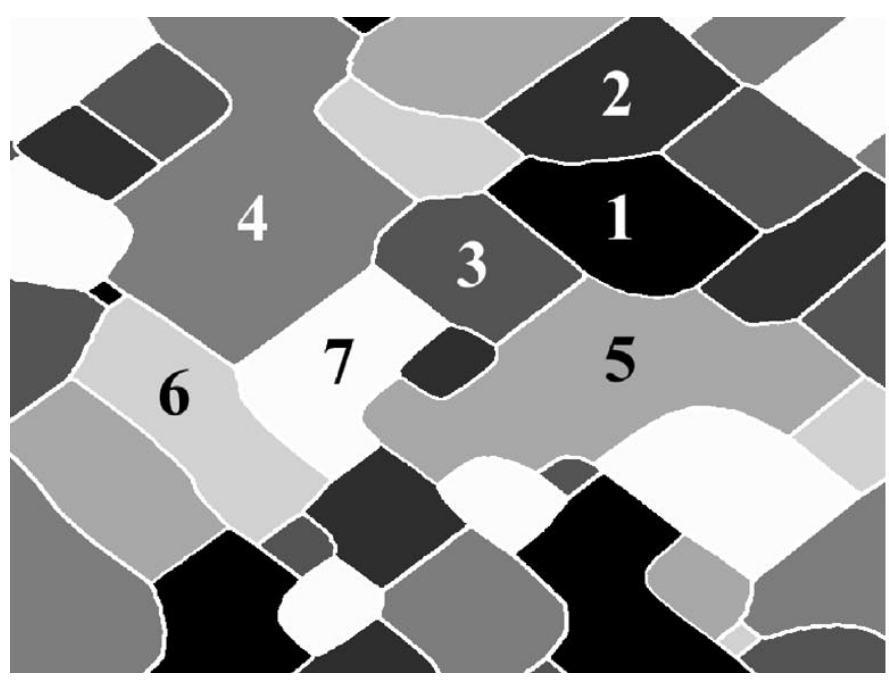

(a)

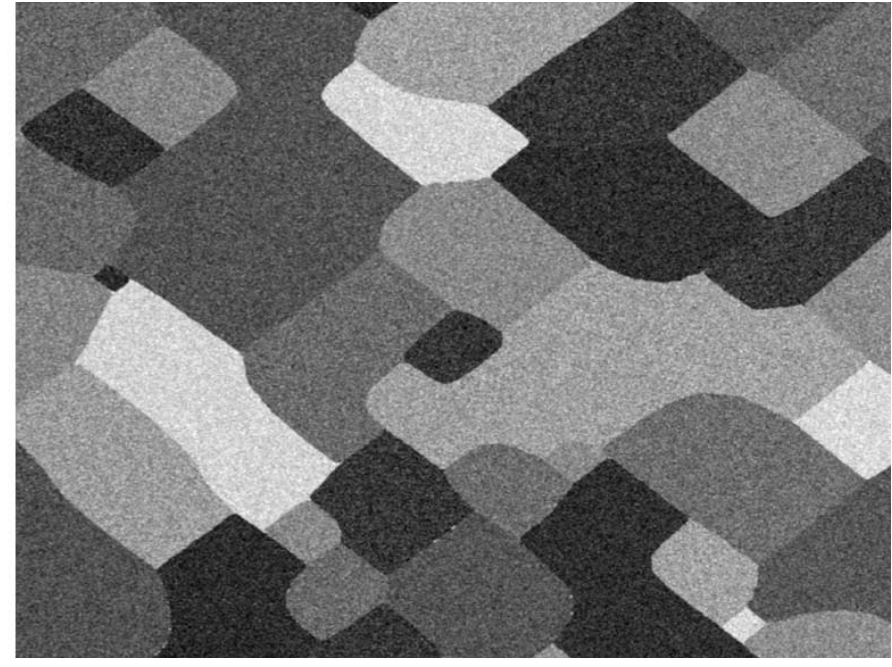

(b)

Fig. 5. Cartoon image and simulated SAR image. (a) Cartoon image (edges overlaid) having seven classes. The seven classes reflect the following crops (from black to white and numbered 1-7): oats, peas, rye, spring barley, winter barley, winter rape, and winter wheat. (b) Simulated polarimetric SAR image (span representation) reflecting the cartoon image and using the parameters from Table I (L-band).

TABLE I

Average Covariance Matrix PARAmeters For SEVEn Crops IN L- AND C-BAND EMISAR ACQUiSitions From May 20

\begin{tabular}{llllllc}
\hline \hline Frequency & Crop & $\sigma_{h h}[\mathrm{~dB}]$ & $\sigma_{h v}[\mathrm{~dB}]$ & $\sigma_{v v}[\mathrm{~dB}]$ & $\left|\rho_{h h, v v}\right|$ & $\angle \rho_{h h, v v}$ [deg.] \\
\hline \multirow{6}{*}{ L-band } & Oats & -22.6 & -32.7 & -22.2 & 0.344 & -161.03 \\
& Peas & -21.8 & -34.9 & -21.9 & 0.688 & 54.07 \\
& Rye & -16.8 & -26.7 & -14.8 & 0.602 & -3.10 \\
& Spring barley & -19.7 & -29.1 & -19.8 & 0.425 & 96.78 \\
& Winter barley & -14.1 & -28.8 & -14.9 & 0.697 & 10.79 \\
& Winter rape & -10.8 & -14.9 & -11.6 & 0.280 & 30.79 \\
& Winter wheat & -17.9 & -29.4 & -18.3 & 0.611 & -72.75 \\
& & & & & \\
& Oats & -10.7 & -18.9 & -13.4 & 0.404 & -4.23 \\
& Peas & -10.0 & -16.7 & -10.6 & 0.408 & 26.87 \\
& Rye & -11.6 & -20.3 & -14.5 & 0.349 & -1.42 \\
& Spring barley & -10.5 & -17.0 & -11.0 & 0.496 & 6.16 \\
& Winter barley & -12.1 & -23.1 & -17.3 & 0.241 & 12.07 \\
& Winter rape & -8.4 & -13.1 & -8.0 & 0.288 & -8.73 \\
& Winter wheat & -12.8 & -21.8 & -18.4 & 0.237 & 10.22 \\
\hline \hline
\end{tabular}

estimated from a number of fields in an L- and C-band EMISAR acquisition, and the seven classes in the two simulated SAR images (denoted the L-band and C-band simulated images) are listed in Table I. A Span-image of the L-band simulated SAR image is shown in Fig. 5(b).

\section{RESULTS AND DISCUSSION}

The polarimetric edge detector is evaluated using simulated polarimetric SAR data as well as polarimetric EMISAR data. The ratio edge detector using single and multiple intensity images is applied for comparison.

\section{A. Distribution of the Test Statistic}

Simulated images ensure totally homogeneous complex Wishart distributed data, and we apply two simulated polarimetric images having a single class (winter barley at L-band and C-band, respectively) for testing the distribution of the test statistic for the polarimetric edge detector. Fig. 6(a) shows the theoretical and observed cumulative distribution functions (cdf) of $-2 \rho \log Q$ as given by (5) for filter configuration
$K_{f}=\{9,3,1, \pi\}$ (i.e., a single orientation) resulting in $L_{f}=90$. Sets of theoretical and observed cdfs are shown for the four cases of fully polarimetric data, azimuthally symmetric data, diagonal data, and two simultaneous images of azimuthally symmetric data. The observed cdfs are shown as points, and an excellent agreement exists between predictions and observations for all four cases.

In Fig. 6(b), we have applied the filter configuration $K_{f}=\{9,3,1, \pi / 4\}$ (i.e., four orientations). Following the filtering scheme from Section III-A, the maximum edge strength $(-2 \rho \log Q)_{\max }$ from the four filters is saved at each pixel. These observed values are compared with the theoretical distribution given by (13). However, the theoretical distribution assumes that $Q_{(i)}$ from the four filters are independent, which is not the case as discussed in Section III-A; hence, we apply a value of $N_{f}$ resulting in the best match with the observations. The four theoretical distributions in Fig. 6(b) representing fully polarimetric data, the azimuthally symmetric case, the diagonal case, and two simultaneous sets of forced azimuthally symmetric data are all made using $N_{f}=1.8$, resulting in a good match between the theoretical and observed distributions 


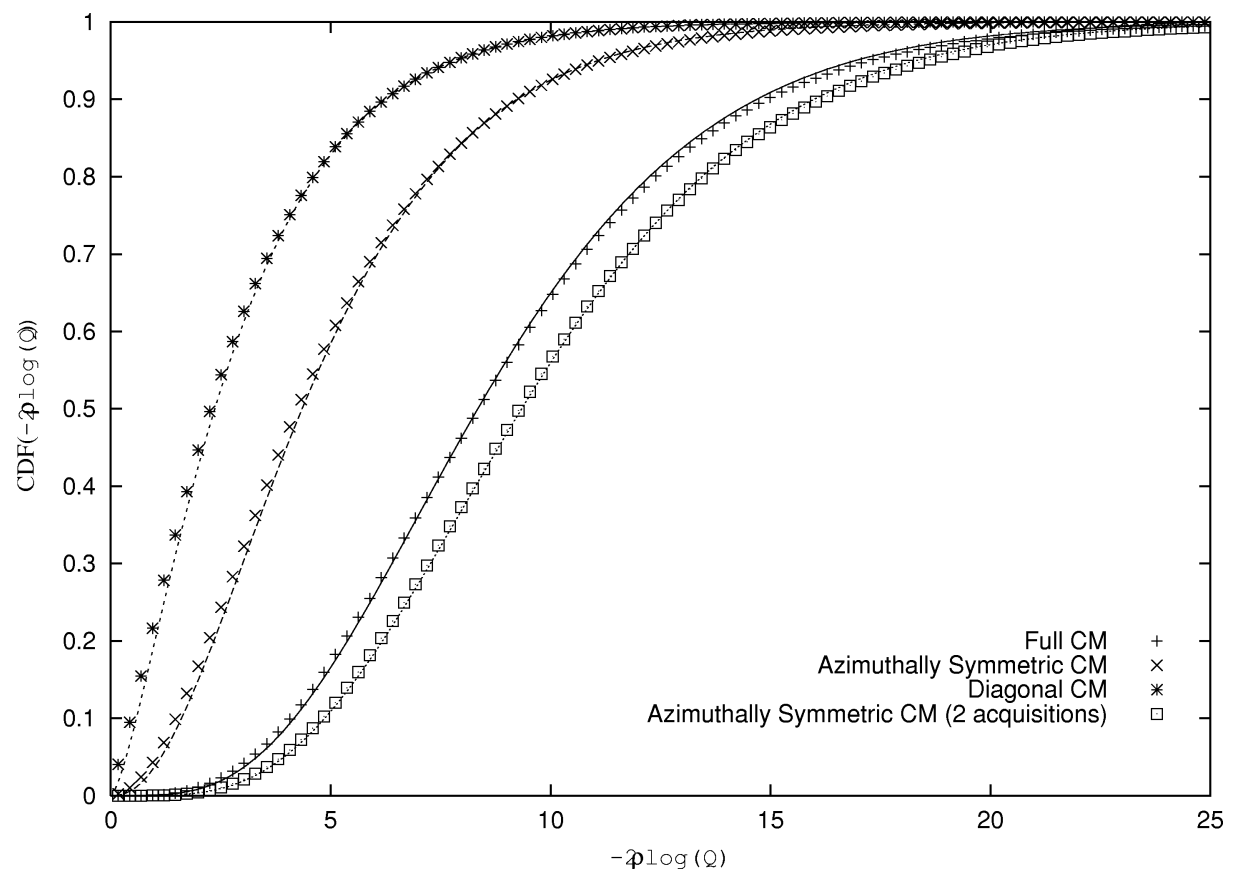

(a)

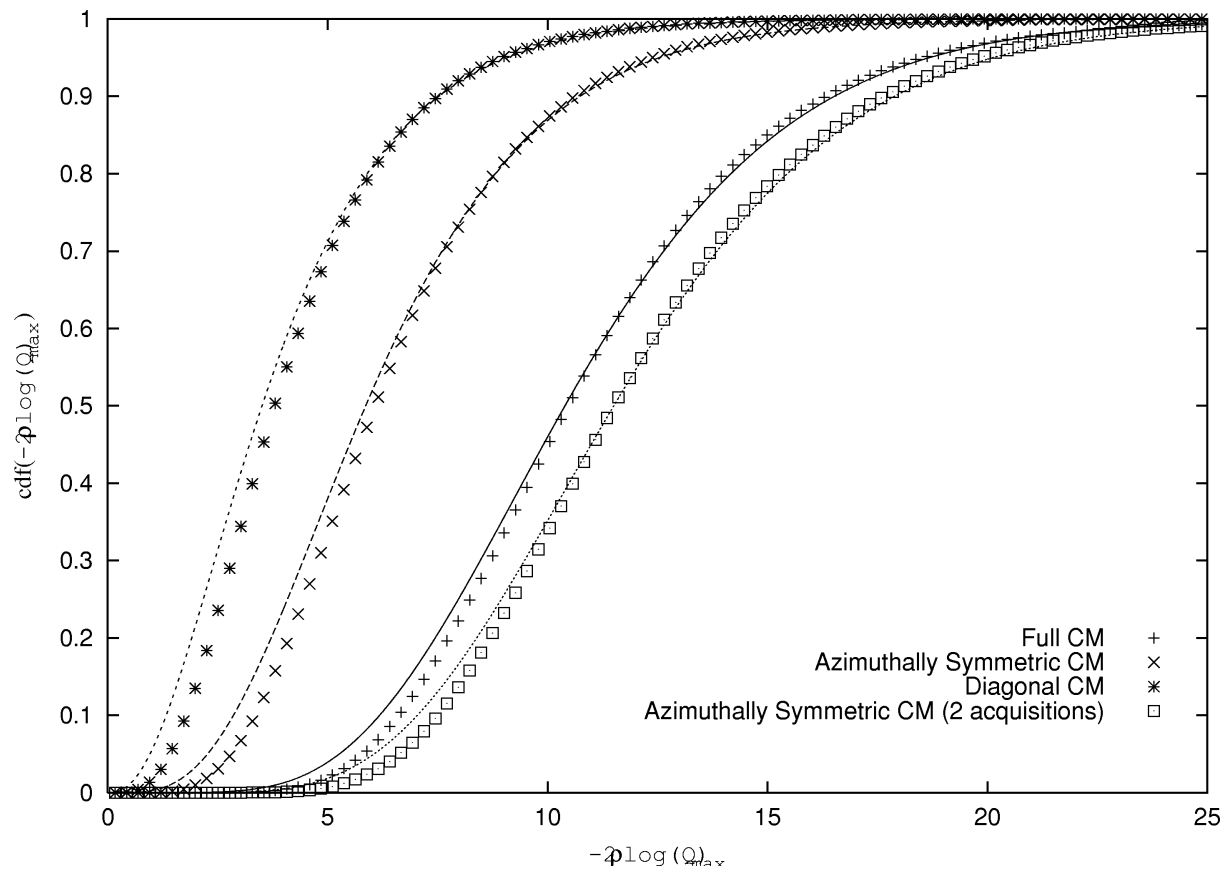

(b)

Fig. 6. CDF of the theoretical and observed distribution of (a) $-2 \rho \log (Q)$ and (b) $(-2 \rho \log (Q))_{\max }$, using filter configurations $K_{f}=\{9,3,1, \pi\}$ and $K_{f}=\{9,3,1, \pi / 4\}$, respectively. The four sets of theoretical and observed cdfs (the observations are the points) accounts for fully polarimetric CM data, forced azimuthally symmetric CM data, forced diagonal CM data, and forced azimuthally symmetric CM data for two images.

for $\operatorname{cdf}\left((-2 \rho \log Q)_{\max }\right) \geq 0.7$, which is normally the region we are interested in when applying the test statistic.

\section{B. Edge Detection Using Simulated SAR Data}

The two simulated polarimetric SAR images using L-band and C-band CM information are now used for testing the polarimetric edge detector in two cases: the case of diagonal sample $\mathrm{CM}$ data and the case of azimuthally symmetric sample CM data. Fig. 7(a) and (b) shows the edge maps when applying the filter configuration $K_{f}=\{9,3,1, \pi / 4\}$ and $P_{\mathrm{fa}, N_{f}}=1 \%$ for the diagonal and azimuthally symmetric case, respectively, using the simulated L-band SAR image from Fig. 5(b). Comparing the edge map in Fig. 7(a) with the cartoon image in Fig. 5(a) we find that the detector using diagonal sample CM data is unable to fully distinguish between the following sets of classes at the specific probability of false alarm: oats and peas, and spring barley and winter wheat. Comparing these combinations with the L-band parameters in Table I, it is verified that the backscattering coefficients are quite similar for oats and peas and for spring barley and winter wheat; hence, we need to apply 


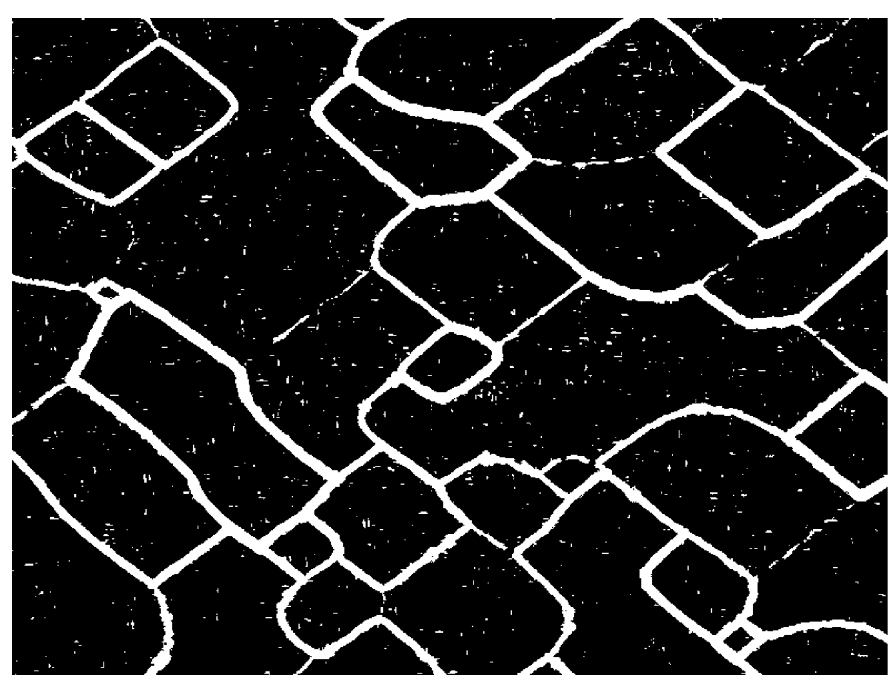

(a)

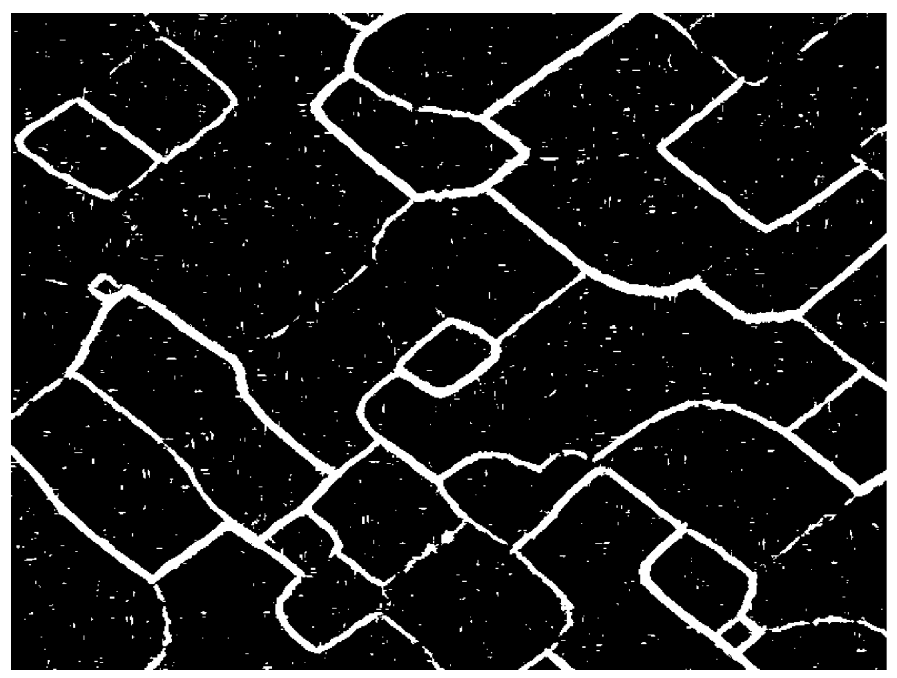

(c)

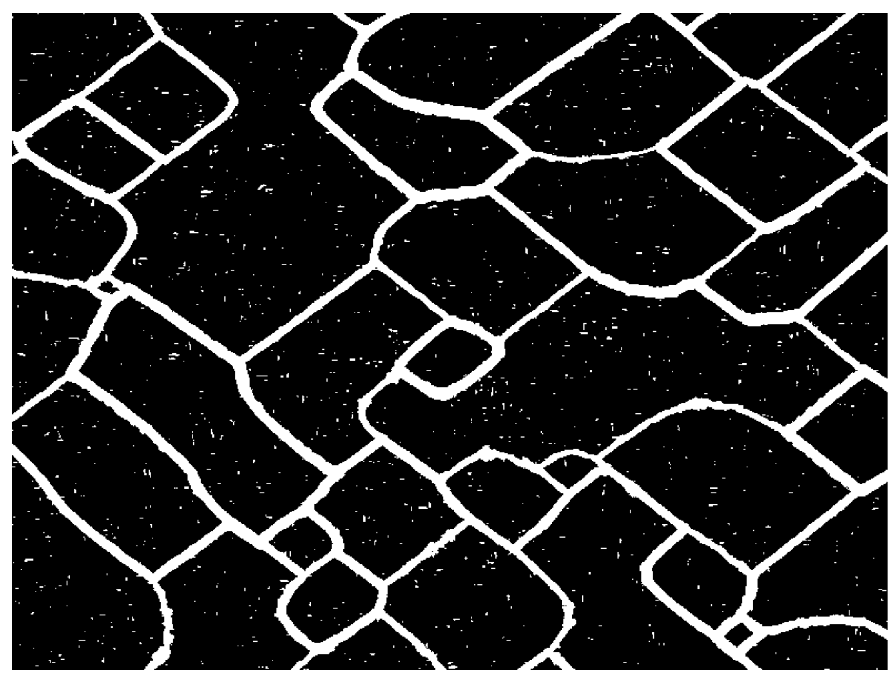

(e)

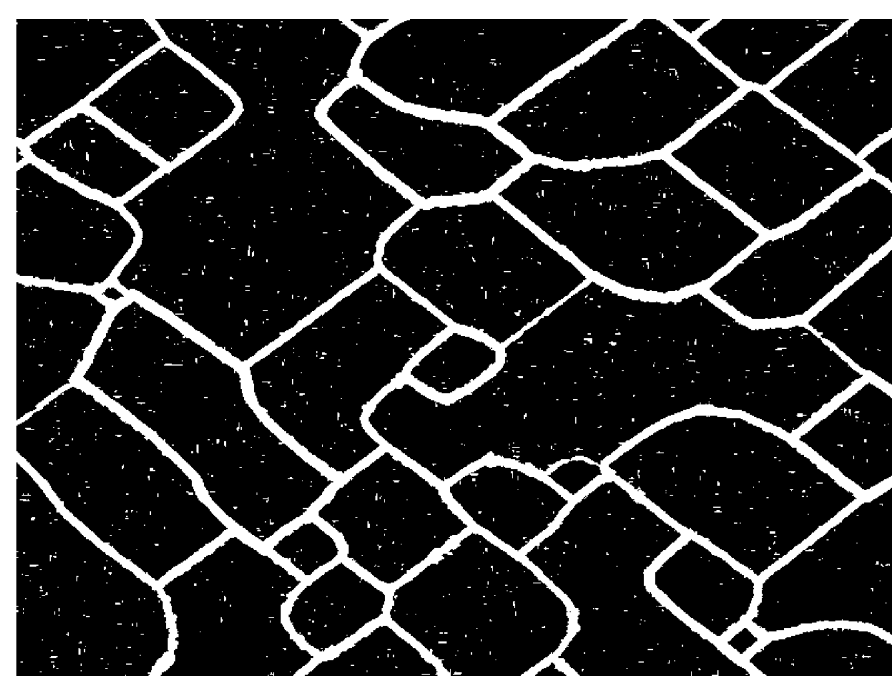

(b)

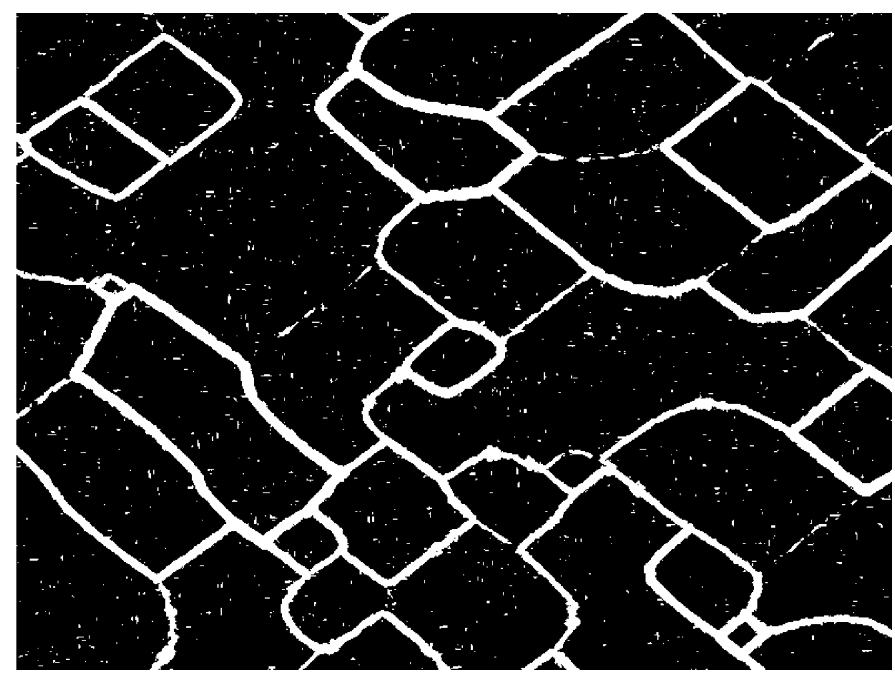

(d)

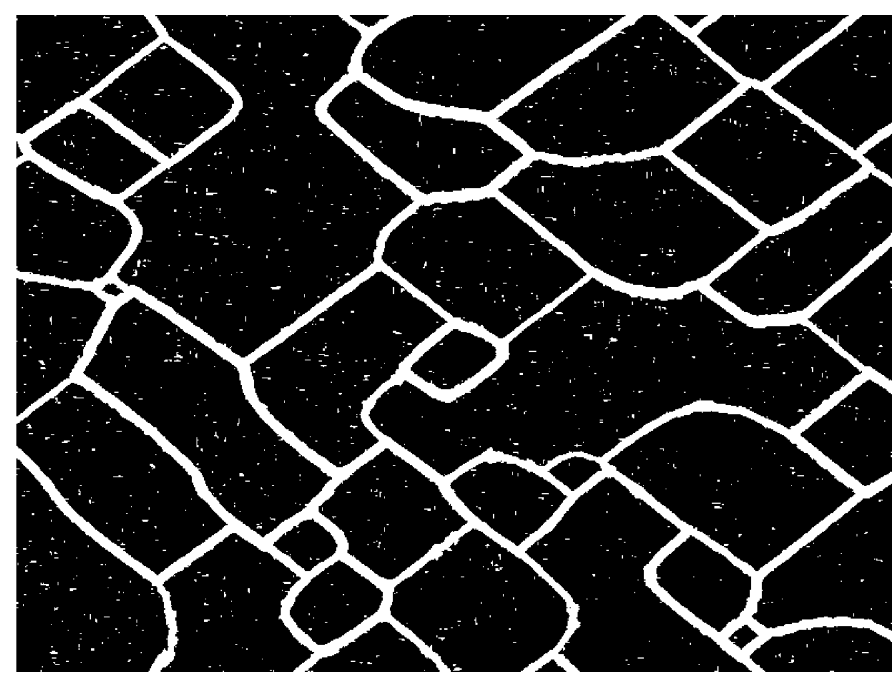

(f)

Fig. 7. Edge maps of simulated polarimetric SAR images using $P_{\mathrm{fa}, N_{f}}=1 \%$. (a) Polarimetric detector using diagonal CM data (L-band), (b) polarimetric detector using azimuthally symmetric CM data (L-band), (c) ratio detector using $\sigma_{\mathrm{hh}}$ (L-band), (d) ratio detector using $\sigma_{\mathrm{hh}}, \sigma_{\mathrm{hv}}$, and $\sigma_{\mathrm{vv}}$ (L-band), (e) polarimetric detector using diagonal CM data $(\mathrm{L}+\mathrm{C}$-band), and (f) polarimetric detector using azimuthally symmetric CM data $(\mathrm{L}+\mathrm{C}$-band).

a larger probability of false alarm (a lower threshold) to be able to distinguish these classes satisfactorily.
The edge map in Fig. 7(b) using the azimuthally symmetric sample CM data appears visually better than when using diag- 
TABLE II

Figure-of-Merit Rating Factors for Edge Maps Based on Simulated Data For the Polarimetric Edge Detector (Azimuthally Symmetric Sample CM Data and Diagonal Sample CM Data) and the Ratio Detector (Single and Multiple Backscatter Images) Using $P_{\mathrm{fa}, N_{f}}=1 \%$

\begin{tabular}{lccc}
\hline \hline Edge detector & L-band & C-band & L+C-band \\
\hline Polarimetric detector (forced azimuthal symmetric) & 0.845 & 0.601 & 0.873 \\
Polarimetric detector (forced diagonal) & 0.763 & 0.639 & 0.851 \\
Ratio detector $\left(\sigma_{h h}, \sigma_{h v}, \sigma_{v v}\right)$ & 0.726 & 0.607 & \\
Ratio detector $\left(\sigma_{h h}\right)$ & 0.595 & 0.215 & \\
Ratio detector $\left(\sigma_{h v}\right)$ & 0.608 & 0.538 & \\
Ratio detector $\left(\sigma_{v v}\right)$ & 0.590 & 0.599 & \\
\hline \hline
\end{tabular}

onal CM data, and all of the actual edges between classes are detected. For both of the edge maps in Fig. 7(a) and (b), it is noticed that several of the edges are quite wide, due to the fact that $\mathbf{Z}_{x}$ and $\mathbf{Z}_{y}$ are estimated in a neighborhood around the central pixel. If thin edges are required, one of the many edge-thinning algorithms suggested in the literature may be applied to the edge map subsequently.

Fig. 7(c) and (d) shows the edge maps when applying the ratio detector to a single backscatter image $\sigma_{\mathrm{hh}}$ and to all three backscatter images using the approach outlined in Section III-B and using the same filter configuration and probability of false alarm as for the polarimetric detector (using two and six effective filters, respectively, in (17) leading to a very good correspondence between predicted and observed cdfs). Clearly, using only the hh polarization results in an edge map where only some of the actual edges are detected, whereas the result using all three backscatter images resembles the result from the polarimetric detector using only the diagonal elements.

The test statistic is also valid in the case of multiple SAR images as discussed in Section II-B. Using the L- and C-band simulated images and the same filter configuration and probability of false alarm as for a single test image, the edge map for the multifrequency diagonal case is shown in Fig. 7(e). It is observed that the combination of L-band and C-band data improves the result for the edges causing problems in the L-band diagonal case (i.e., between oats and peas and spring barley and winter wheat). This is due to the fact that the fields have different scattering mechanisms at L- and C-band; hence, the probability of observing an edge between neighboring fields is generally increased when applying multiple datasets in the detection. For the azimuthally symmetric case, the edge map using only L-band appeared visually very good, and only a small improvement is seen in Fig. 7(f).

Like Fjørtoft et al. [3], we apply the figure-of-merit rating factor $R$ suggested in [23] for a more quantitative evaluation of the edge maps. Denoting the number of ideal and detected edge map points as $N_{i}$ and $N_{d}$, respectively, $R$ is given as

$$
R=\frac{1}{\max \left(N_{i}, N_{d}\right)} \sum_{j=1}^{N_{d}} \frac{1}{1+\alpha d_{j}^{2}}
$$

where $\alpha$ is a scaling parameter (set to one in this paper), and $d_{j}$ is the minimum distance of detected edge point $j$ to an ideal edge point. The distances $d_{j}$ are obtained by applying a Chamfer-11.3507 distance transformation to the ideal edge map providing the distance map [24]. The ideal edge map consists of all pixels having a Euclidean distance to the boundary between two different regions smaller than or equal to five; this set of ideal edge pixels is chosen, since an edge may be detected five pixels away from the actual edge using the applied filter configuration $K_{f}=\{9,3,1, \pi / 4\}$. The rating factor belongs to the interval $[0,1]$, with $R=1$ corresponding to the ideally detected edge map.

Table II shows the rating factors for the polarimetric detector when applying azimuthally symmetric CM data and diagonal $\mathrm{CM}$ data, respectively, from the L- and C-band simulated images. Also, the results for the ratio detector when applying single intensity images as well as all three intensity images are shown. The rating factors for the L-band test image supports the previous discussion, i.e., the polarimetric detector using azimuthally symmetric sample CM data provides the overall best edge map. For the C-band test image, the diagonal case actually performs better than the azimuthally symmetric case; in Table I, it is seen that the argument and phase of the complex correlation coefficient has smaller differences between the classes in C-band than in L-band; hence, the addition of this term in the $\mathrm{CM}$ is less useful for edge detection in C-band. The overall best rating factors are obtained using multifrequency polarimetric edge detection with the azimuthally symmetric case performing slightly better than the diagonal case.

If only the diagonal terms of the $\mathrm{CM}$ are available, the polarimetric detector and the ratio detector (using all three terms) provides results in the approximately same range. The ratio detector using a single backscatter image is more sensitive to the applied images, resulting in very variable performance. This effect is especially noticeably for $\sigma_{\mathrm{hh}}$, where the low contrast between classes for the C-band image results in a very low rating factor compared to the L-band image.

\section{Edge Detection Using EMISAR Images}

The polarimetric edge detector is applied to the test area shown in Fig. 4 for the case of L-band azimuthally symmetric and diagonal sample $\mathrm{CM}$ data. A single filter configuration $K_{f}=\{9,3,1, \pi / 4\}$ has been used, and the edge maps are shown in Fig. 8(a) and (b), respectively. The edge maps have been scaled between the threshold values $T_{f}$ corresponding to $P_{\mathrm{fa}, N_{f}}=1 \%$ and $3 \times T_{f}$. It is observed that two significant edges are missing or appear very weak in the diagonal case, i.e., between a winter wheat and a spring barley field (denoted A and B, respectively, in Fig. 4) and between an oat field and a pea field (denoted C and D, respectively, in Fig. 4). Both 


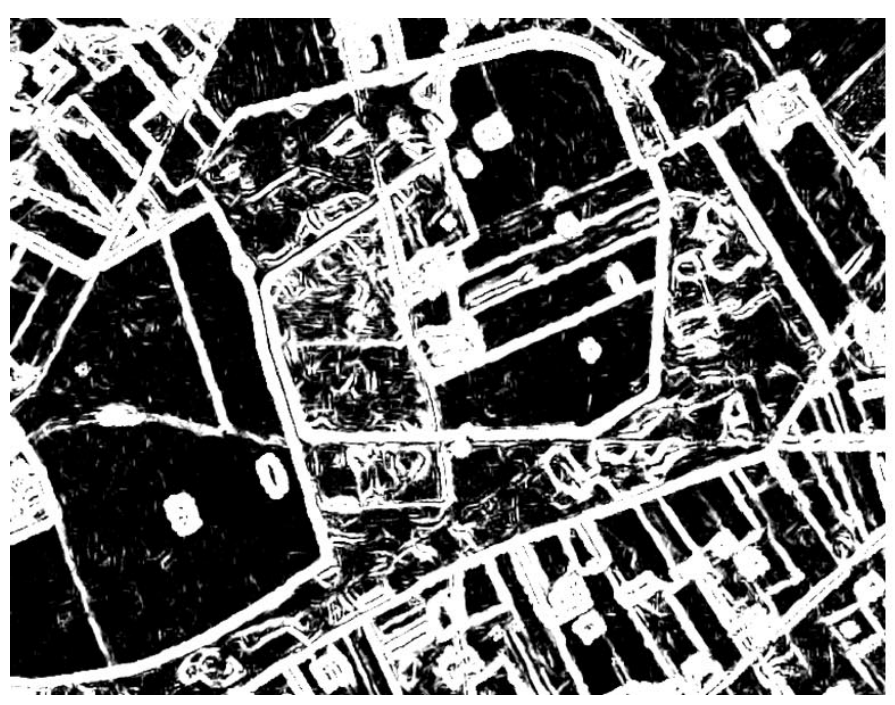

(a)

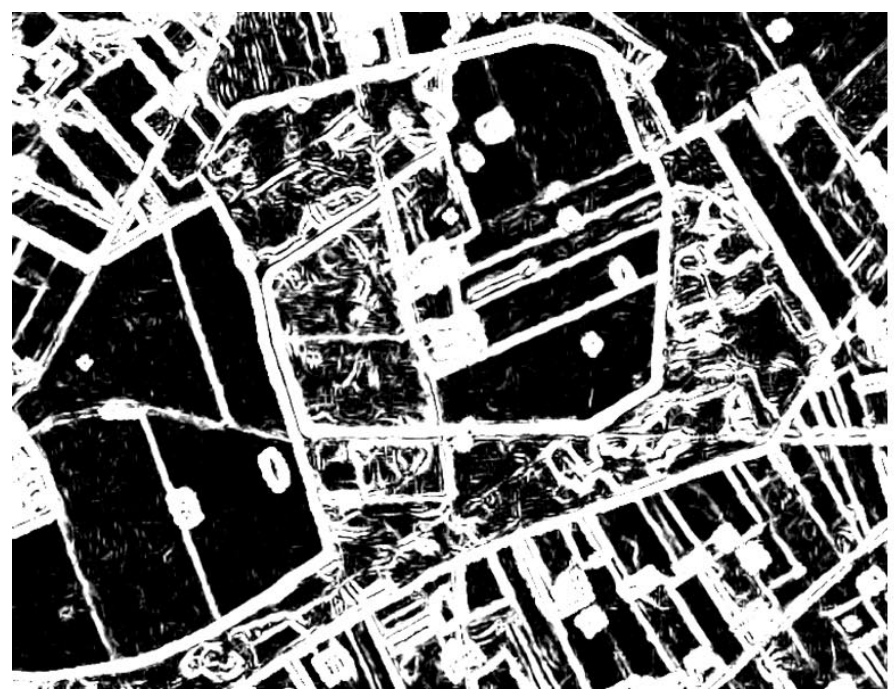

(c)

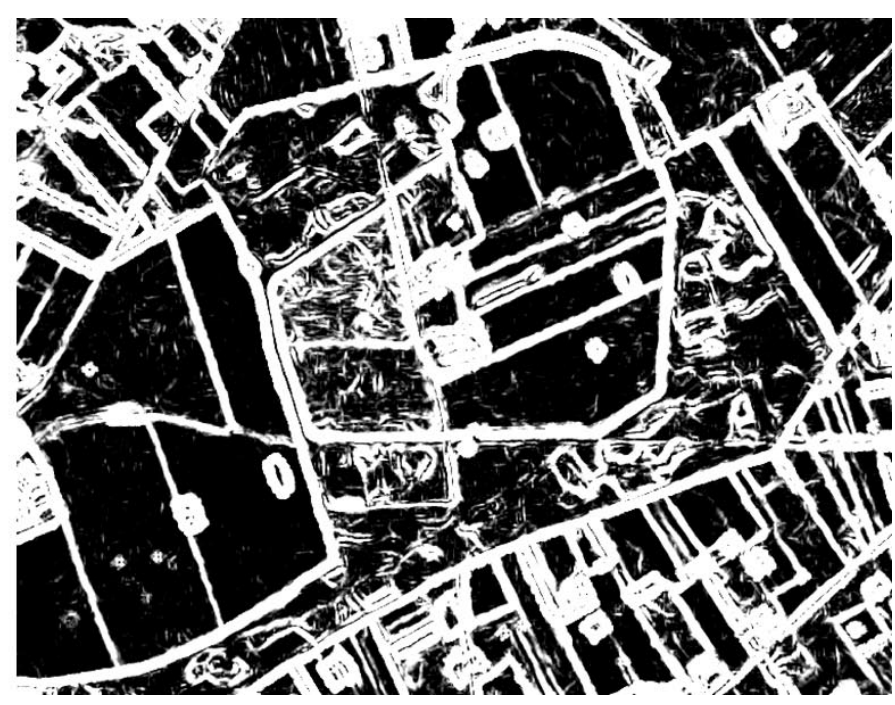

(b)

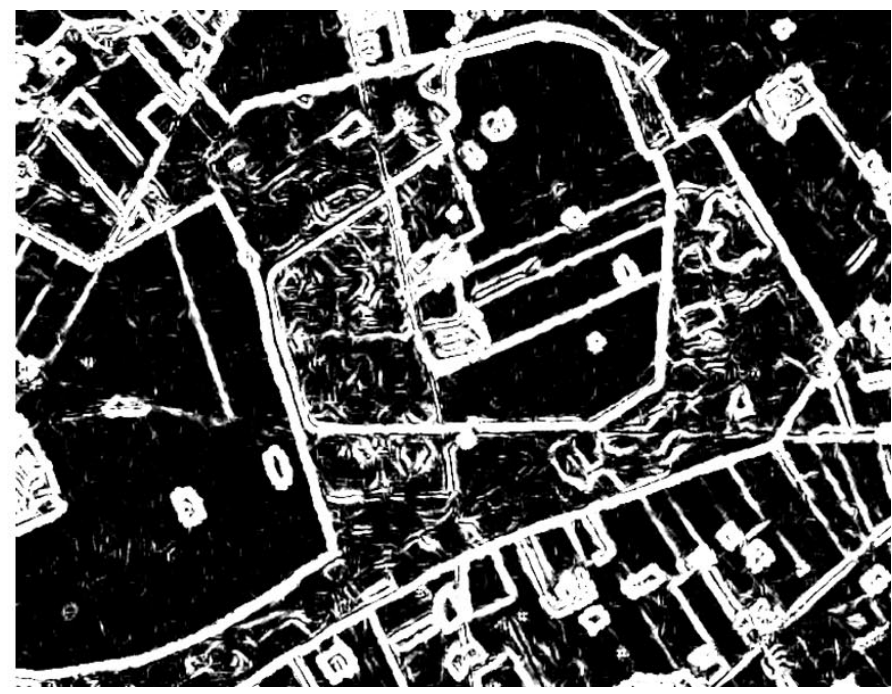

(d)

Fig. 8. Scaled edge maps of test area from Fig. 4 using filter configuration $K_{f}=\{9,3,1, \pi / 4\}$. (a) Polarimetric detector using diagonal CM data (L-band), (b) polarimetric detector using azimuthally symmetric CM data (L-band), (c) polarimetric detector using diagonal CM data (L + C-band), and (d) ratio detector using $\sigma_{\mathrm{hh}}$ (L-band).

of these missing edges are expected when comparing with Fig. 7(a), where the same two combinations of fields caused problems as well. On the other hand, both edges are clearly detected in the case of azimuthally symmetric sample CM data, as we also expect from Fig. 7(b).

Fig. 8(c) shows the edge map when applying L- and C-band diagonal sample $\mathrm{CM}$ data using the same filter configuration and probability of false alarm. The edge between the winter wheat and spring barley field is now clearly detected, primarily due to the large difference between $\sigma_{\mathrm{hv}}$ and $\sigma_{\mathrm{vv}}$ for the two crops at $\mathrm{C}$-band, as seen in Table I. The edge between the oat field and the pea field is still not detected; this is not unexpected when comparing with Fig. 7(e), where the edge between the two crops is less significant than the other edges in the edge map. Also, in Table I shows that the backscattering coefficients for the two crops are in the same range at C-band as well as L-band.

Finally, Fig. 8(d) shows the edge map when applying the standard ratio detector to a single intensity image $\sigma_{\mathrm{hh}}$ at L-band using the same filter configuration and probability of false alarm. It is clearly observed how several of the edges between the fields are missing in the resulting edge map.

The results in Fig. 8 using real SAR data illustrate that the polarimetric information in the complex correlation coefficient can, in fact, improve the edge maps. Also, the application of multifrequency (and multitemporal) SAR data may improve the edge detection results.

\section{CONCLUSION}

In this paper, a new edge detector for polarimetric SAR data is presented. Based on a test for equality of covariance matrices, edges are detected by comparing covariance matrices on each side of the pixel of interest. Using an associated asymptotic distribution of the test statistic, the edge detection can be performed at a given probability of false alarm, and as the asymptotic distribution is independent of the parameters contained in the CM, the edge detector belongs to the class of constant CFAR detectors, making it suitable for SAR images. 
An important property of the polarimetric edge detector is that it can be applied to a wide range of SAR data, i.e., full covariance matrix data, forced azimuthally symmetric covariance matrix data, and forced diagonal covariance matrix data, but also single-channel backscattering images and multitemporal and/or multifrequency polarimetric data. This enables the simultaneous processing of, for example, fully polarimetric airborne SAR data and spaceborne single-channel SAR data. By simply changing the parameters in the asymptotic distribution accordingly, CFAR detection is obtained for all of these data.

An adaptive filtering scheme is outlined, and a procedure for obtaining the thresholds associated with a given probability of false alarm is described. When applying filters oriented at more than one orientation, an approximate relation between the observed and predicted distributions of the test statistic is provided. The relation behaves as expected when applied to simulated polarimetric data.

Applying the polarimetric edge detector to a simulated test image results in improved edge maps when using azimuthally symmetric CM data compared to using only diagonal CM data. This improvement is also observed for polarimetric EMISAR data.

\section{ACKNOWLEDGMENT}

The authors wish to thank J. M. Carstensen (Informatics and Mathematical Modeling, Technical University of Denmark) for providing the source code for the Potts model [25].

\section{REFERENCES}

[1] M. Bennamoun, "Edge detection: Problems and solutions," in Proc. IEEE Int. Conf. Systems, Man Cybernetics, vol. 4, 1997, pp. 3164-3169.

[2] R. Touzi, A. Lopès, and P. Bousquet, "A statistical and geometrical edge detector for SAR images," IEEE Trans. Geosci. Remote Sensing, vol. 26, pp. 764-773, Nov. 1988.

[3] R. Fjørtoft, A. Lopès, P. Marthon, and E. Cubero-Castan, "An optimal multiedge detector for SAR image segmentation," IEEE Trans. Geosci. Remote Sensing, vol. 36, pp. 793-802, May 1998.

[4] S. N. Madsen, "Speckle theory: Modeling, analysis and applications related to synthetic aperture radar data," Ph.D. thesis, Dept. of Electromagnetic Systems, Tech. Univ. Denmark, Lyngby, Denmark, 1986.

[5] A. Lopes, E. Nezry, R. Touzi, and H. Laur, "Structure detection and statistical adaptive speckle filtering in SAR images," Int. J. Remote Sens., vol. 14, no. 9, pp. 1735-1758, 1993.

[6] H. Skriver, "Extraction of sea ice parameters from synthetic aperture radar images," Ph.D. thesis, Dept. of Electromagnetic Systems, Tech. Univ. Denmark, Lyngby, Denmark, 1989.

[7] A. Baraldi and F. Parmiggiani, "Refined gamma MAP SAR speckle filter with improved geometrical adaptivity," IEEE Trans. Geosci. Remote Sensing, vol. 33, pp. 1245-1257, Sept. 1995.

[8] F. Tupin, H. Maitre, J.-F. Mangin, J.-M. Nicolas, and E. Pechersky, "Detection of linear features in SAR images: Application to road network extraction," IEEE Trans. Geosci. Remote Sensing, vol. 36, pp. 434-453, Mar. 1998.

[9] C. J. Oliver, D. Blacknell, and R. G. White, "Optimum edge detection in SAR," Proc. Inst. Elect. Eng., vol. 143, no. 1, pp. 31-40, Feb. 1996.

[10] C. J. Oliver and P. Lombardo, "Simultaneous mean and texture edge detection in SAR clutter," Proc. Inst. Elect. Eng., vol. 143, no. 6, pp. 391-399, Dec. 1996.

[11] J. J. van Zyl and F. T. Ulaby, "Scattering matrix representation for simple targets," in Radar Polarimetry for Geoscience Applications, F. T. Ulaby and C. Elachi, Eds. Norwood, MA: Artech House, 1990.

[12] J. Schou, W. Dierking, and H. Skriver, "Tensor based structure estimation in multi-channel images," in Proc. IGARSS, vol. 2, 2000, pp 663-665.
[13] R. G. Caves, I. McConnell, R. Cook, and S. Quegan, "Multi-channel SAR segmentation: Algorithms and applications," IEE Colloq. Image Processing for Remote Sensing, no. 5, pp. 2/1-2/6, 1996.

[14] J. S. Lee, M. R. Grunes, and G. de Grandi, "Polarimetric SAR speckle filtering and its implication for classification," IEEE Trans. Geosci. Remote Sensing, vol. 37, pp. 2363-2372, Sept. 1999.

[15] K. Conradsen, A. A. Nielsen, J. Schou, and H. Skriver, "A test statistic in the complex Wishart distribution and its application to change detection in polarimetric SAR data," IEEE Trans. Geosci. Remote Sensing, vol. 41, Jan. 2003, to be published.

[16] N. R. Goodman, "Statistical analysis based on a certain multivariate complex gaussian distribution (an introduction)," Ann. Math. Stat., vol. 34, pp. 152-177, 1963.

[17] M. R. Borgeaud, T. Shin, and J. A. Kong, "Theoretical models for polarimetric radar clutter," J. Electromagn. Waves Appl., vol. 1, pp. 73-89, 1987.

[18] K. Conradsen, En Introduktion Til Statistik. Lyngby, Denmark: IMSOR, Tech. Univ. Denmark, 1984.

[19] E. L. Christensen, N. Skou, J. Dall, K. W. Woelders, J. H. Jrgensen, J. Granholm, and S. N. Madsen, "EMISAR: An absolutely calibrated polarimetric L- and C-band SAR," IEEE Trans. Geosci. Remote Sensing, vol. 36, pp. 1852-1865, Nov. 1998.

[20] W. Dierking, J. Schou, and H. Skriver, "Change detection of small objects and linear features in multi-temporal polarimetric images," in Proc. IGARSS, 2000, pp. 1693-1696.

[21] J. Besag, "On the statistical analysis of dirty pictures," J. R. Stat. Soc., ser. B, vol. 48, no. 3, pp. 259-302, 1986.

[22] L. M. Novak and M. C. Burl, "Optimal speckle reduction in polarimetric SAR imagery," IEEE Trans. Aerosp. Electron. Syst., vol. 26, pp. 293-305, Mar. 1990.

[23] W. K. Pratt, Digital Image Processing. New York: Wiley, 1978.

[24] G. Borgefors, "Distance transformations in arbitrary dimensions," Comput. Vision, Graph. Image Processing, vol. 27, no. 3, pp. 321-345, 1984.

[25] J. M. Carstensen, "Description and simulation of visual texture," Ph.D. thesis, IMSOR, Tech. Univ. Denmark, Lyngby, Denmark, 1992.

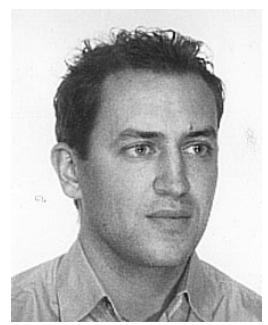

Jesper Schou received the M.S. degree in engineering and the Ph.D. degree in electrical engineering from the Technical University of Denmark, Lyngby, Denmark, in 1997 and 2001 respectively. His primary research interests during his Ph.D. studies was image analysis and the processing of polarimetric SAR data, including filtering, structure detection, and segmentation.

$\mathrm{He}$ is currently working with 3-D scanner systems and related 3-D image analysis algorithms.

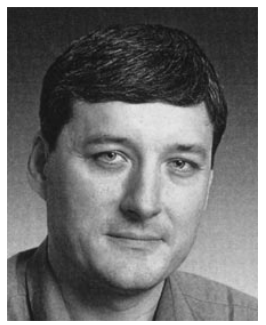

Henning Skriver received the M.S. degree and the Ph.D. degree from the Technical University of Denmark, Lyngby, in 1983 and 1989, respectively, both in electrical engineering.

He has been with the Section of Electromagnetic Systems (EMI), Department Ørsted, Technical University of Denmark (DTU), Lyngby, since 1983, where he is currently an Associate Professor. His work has been concerned primarily with various topics related to the utilization of SAR data for different applications. From 1983 to 1992, his main area of interest was retrieval of sea ice parameters from SAR data, including SAR data from ERS-1. Since 1992, he has covered different aspects of land applications of SAR data, such as forestry in the MAESTRO-1 project, and agricultural and environmental applications using both satellite SAR data and data from the Danish airborne polarimetric SAR, EMISAR. His interests also include various methods for processing of SAR data, such as SAR image simulation, SAR image filtering, speckle statistics, texture analysis, segmentation, calibration, and polarimetric analysis. 


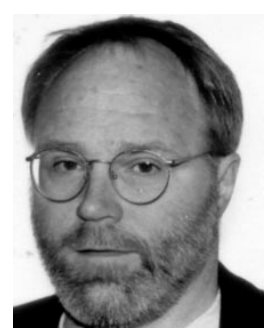

Allan Aasbjerg Nielsen received the M.S. degree from the Department of Electrophysics, Technical University of Denmark, Lyngby, Denmark, in 1978, and the Ph.D. degree from Informatics and Mathematical Modelling (IMM), Technical University of Denmark, in 1994.

$\mathrm{He}$ is currently an Associate Professor with IMM. He is currently working with IMM's Section for Geoinformatics. He has been with the Danish Defense Research Establishment from 1977 to 1978. He has worked on energy conservation in housing with the Thermal Insulation Laboratory, Technical University of Denmark, from 1978 to 1985 . Since 1985, he has been with the Section for Image Analysis, IMM. Since then, he has worked on several national and international projects on the development, implementation, and application of statistical methods and remote sensing in mineral exploration, mapping, geology, environment, oceanography, geodesy, and agriculture funded by industry, the European Union, Danida (the Danish International Development Agency), and the Danish National Research Councils.

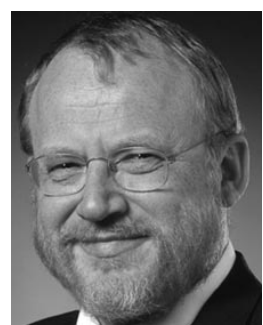

Knut Conradsen received the M.S. degree from the Department of Mathematics, University of Copenhagen, Copenhagen, Denmark, in 1970.

$\mathrm{He}$ is currently a Professor with the Informatics and Mathematical Modelling, Technical University of Denmark (DTU), Lyngby, Denmark. Since 1995, he has been Deputy Rector (or Vice President) of DTU. His main research interest is the application of statistics and statistical models to real-life problems. He has worked on many national and international projects on the application of statistical methods in a wide range of applications, recently mainly in remote sensing. He has also conducted extensive studies in the development and application of mathematical and statistical methods concerning spatial, multi/hyperspectral, and multitemporal data, including both optical and radar data. 